Disclosures S. Raymond: None. T. Leslie-Mazwi: None. N. Rost: None. P. Schaefer: None. J. Hirsch: 2; C; Medtronic. R. Gonzalez: None. J. Rabinov: None.

\section{E-040 FIVE-YEAR SINGLE CENTER EXPERIENCE OF INTRACRANIAL ANEURYSM TREATMENT WITH THE PED IN PATIENTS OF DIFFERENT AGE GROUPS}

A Kuhn, J Lozano, K de Macedo Rodrigues, F Massari, A Wakhloo, D Rex, M Gounis, M Marosfoi, M Perras, C Brooks, M Howk, A Puri. Division of Neuroimaging and Intervention, Department of Radiology and New England Center for Stroke, University of Massachusetts, Worcester, MA

\subsection{6/neurintsurg-2016-012589.112}

Purpose To evaluate safety and efficacy of the pipeline embolization device (PED) in patients of different age groups and with incidentally found or recanalised (previously coiled or clipped) aneurysms.

Materials and methods All patients with an incidentally found or recanalised aneurysm and treated with the PED at our institution between 2011 and 2016 were included. We then divided the patient cohort into three age groups representing young ( $\mathrm{Y}, \leq 45$ years), middle age $(\mathrm{M}, 46-<65$ years) and older $(\mathrm{O}, \geq 65$ years $)$ individuals. Information on patient's vascular risk factors, presenting symptoms and mRS on admission was collected. Follow-up imaging was evaluated for aneurysmal occlusion and the presence of intimal hyperplasia. Patient clinical outcome at discharge, 6 and 12 months was documented.

Results We included 140 patients harboring 164 aneurysms with 20 patients in the young age group, 88 in the middle age and 32 in the older age group. Male to female ratio was approximately 1:4 overall and interestingly in all age groups as well. The majority of aneurysms were located in the anterior circulation (94.5\%) and found incidentally (75\%). Twenty-four aneurysms had been previously treated (12 ruptured and 12 unruptured). Smoking, hypertension and dyslipidemia were the most frequently encountered vascular risk factors in all age groups, with smoking being most common in the young, hypertension the most common in the middle age and hypertension/dyslipidemia the most common in the older age group. Median mRS on admission and discharge was 0 for all age groups. The median mRS remained 0 at 6 and 12 month follow-up. Overall mortality and morbidity rate was $2.1 \%(3 / 140, M=2$ and $\mathrm{O}=1)$, Complete aneurysm occlusion at 6 and 12 months was seen in 77\% (78/101) and $80 \%$ (52/65), respectively. Mild intimal hyperplasia was seen in 18 cases total $(18 \%)$ with 2 cases in the young, 11 in the middle age and 5 in the older age group. Moderate and severe intimal hyperplasia was found in one case each (young and older age group). No retreatment was required.

Nine aneurysms $(\mathrm{Y}=3, \mathrm{M}=5$ and $\mathrm{O}=1)$ which demonstrated near complete occlusion at 6 months showed complete occlusion in 2 cases $(M=2)$ and stable near complete occlusion in the remaining 7 at the 12 month mark. For aneurysms demonstrating partial occlusion at 6 months $(\mathrm{Y}=2, \mathrm{M}=5, \mathrm{O}=3), 12$ months follow-up showed progression to complete or near complete occlusion in 1 case each $(\mathrm{Y}=1$ and $\mathrm{M}=1)$ and stable partial occlusion in 3 cases $(\mathrm{M}=3)$.

Available 12 months follow-up for mild intimal hyperplasia detected at 6 months showed resolution in 4 cases $(M=1$ and $\mathrm{O}=3$ ) and stable mild hyperplasia in 5 cases $(\mathrm{Y}=1$ and $\mathrm{M}=4)$. One case of moderate intimal hyperplasia at 6 months (young age group) improved to mild hyperplasia at 12 months follow-up.

Subanalysis of treatment outcome of incidentally found aneurysms compared to unruptured pretreated or ruptured pretreated aneurysms showed no difference of flow diverter performance between the different aneurysm groups or among the different patient age groups.

Conclusion PED placement is feasible and safe in patients of different age groups and with incidental or recanalised aneurysms.

Disclosures A. Kuhn: None. J. Lozano: None. K. de Macedo Rodrigues: None. F. Massari: None. A. Wakhloo: 1; C; NIH, Philips Healthcare, Wyss Institute. 2; C; Codman Neurovascular and Stryker Neurovascular. 3; C; Harvard Postgraduate Course, Miami Cardiovascular Institute. 4; C; InNeuroCo Inc, EpiEB and Pulsar Medical. D. Rex: None. M. Gounis: 1; C; $\mathrm{NIH}$, Medtronic Neurovascular, Microvention/Terumo, Cerevasc LLC, Gentuity, Codman Neurovascular, Philips Healthcare, Stryker Neurovascular, Tay Sachs Foundation, and InNeuroCo Inc. 2; C; Codman Neurovascular and Stryker Neurovascular. 4; C; InNeuroCo Inc. M. Marosfoi: None. M. Perras: None. C. Brooks:None. M. Howk: None. A. Puri: 1; C; Stryker Neurovascular and Covidien. 2; C; Codman Neurovascular, Stryker Neurovascular and Covidien. 3; C; Miami Cardiovascular Institute. 4; C; InNeuroCo Inc.

\section{E-041 ENDOVASCULAR TREATMENT OF INTRACRANIAL ANEURYSMS WITH BARRICADE COILS: SAFETY AND EFFICACY IN A PROSPECTIVE SERIES}

L Pierot, M Zidan, C Foussier, G Metaxas, S Soize. Radiology, Hôpital Maison-Blanche, Reims, France

\subsection{6/neurintsurg-2016-012589.113}

Purpose The superiority of endovascular treatment versus surgery in the management of intracranial aneurysms was initially demonstrated in ISAT, endovascular treatment being performed using bare platinum coils. Despite the appearance of new endovascular techniques (stenting, flow diversion, flow disruption), coiling still is the first-line treatment singularly for ruptured aneurysms. New coils are usually not evaluated except if they are surface-modified. However as new bare coils have also different characteristics, it is important to evaluate their safety and efficacy.

Materials and methods Patients with intracranial aneurysms treated between October 2013 and December 2015 in Reims University Hospital by simple coiling or balloon-assisted coiling with Barricade Coils (Blockade Medical, Irvine, California, USA) were prospectively included in a database and retrospectively studied. Patients treated with other devices (stents, flow diverters, flow disrupters) were not included in these series. For all included patients, medical charts, imaging studies and initial and follow-up imaging examinations were reviewed by an independent practitioner that made a comprehensive evaluation of the procedural and post-procedural complications, morbidity and mortality rates, one month clinical follow-up, and anatomical results.

Results From October 2013 to December 2015, 98 patients having 110 saccular intracranial aneurysms were treated with Barricade coils (Blockade Medical, Irvine, California, USA). Ten patients with 13 aneurysms adjunctive devices and were 
excluded. Finally 88 patients $(59$ females, $67.1 \%$ and 29 males, 32.9\%) aged 30 to 83 years (mean: $52 \pm 13$ years) with 97 aneurysms (57 ruptured, 58.7\% and 40 unruptured, 41.3\%) were included. Aneurysm locations were internal carotid artery (36 aneurysms, 30.9\%), anterior communicating and anterior cerebral arteries (31 aneurysms, 31.9\%), middle cerebral artery (16 aneurysms, 16.5\%), vertebrobasilar system (14 aneurysms, 14.4\%). Seventy-seven aneurysms (79.4\%) were treated with simple coiling and 20 (20.6\%) with balloonassisted coiling.

Aneurysm coiling was feasible in all aneurysms. Thromboembolic events with or without clinical worsening were encountered in $14 / 97$ procedures (14.4\%) intraoperative rupture in 6 aneurysms (6.6\%), all without clinical worsening. Overall the procedural morbidity and mortality were encountered in 2 patients $(2.1 \%)$ and 1 patient (1.0\%), respectively.

Post-operative anatomical results were complete aneurysm occlusion in 79 aneurysms (81.4\%), neck remnant in 13 aneurysms (13.4\%) and aneurysm remnant in 5 aneurysms (5.2\%).

Conclusion Preliminary results in this prospective series show good safety and efficacy of Barricade coils in aneurysm treatment.

Disclosures L. Pierot: 2; C; Blockade. M. Zidan: None. C. Foussier: None. G. Metaxas: None. S. Soize: None.

\section{E-042 EFFICACY OF AN AVM CLASSIFICATION SYSTEM THAT DIRECTS ENDOVASCULAR THERAPIES ACCURATELY}

W Yakes. Vascular Malformation Center, Englewood, CO

10.1136/neurintsurg-2016-012589.114

Purpose To determine if AVM angioarchitecture characteristics can be predictive and direct specific curative endovascular procedures accurately and consistently to treat high-flow malformations.

Materials and methods Angiographic analysis of high-flow vascular malformations determined 4 major angioarchitectures. Type I: Direct arterial/arteriolar to vein/venule connection; e. g., as commonly seen in pulmonary AVF, congenital renal AVF, etc. Type II: Arterial/arteriolar connections to a "nidus" that then have several out-flow veins with no intervening capillary beds in any of the vascular interconnections. Type
IIIa: Arterial/arteriolar connections to an aneurysmal vein ("nidus" is the vein wall) that drains into a dominant out-flow vein with no intervening capillary bed in these connections. Type IIIb: Same angioarchitecture as Type IIIa, except that there are more than one (several) out-flow veins. Type IV: "Infiltrative" form of AVM whereby innumerable micro-arteriolar branches fistulize through a tissue (e.g., ear) totally infiltrating it, shunting into multiple out-flow veins. Capillary beds also exist in the tissue and are mixed with the innumerable AVFs. Without the capillaries the tissue could not be viable, therefore must be present.

Results Type I: Can be effectively treated with mechanical devices; e.g., coils, Amplatzer Plugs, etc. Type II: Can be effectively treated with ethanol embolization. Type IIIa: Can be effectively treated by transcatheter ethanol, retrograde vein catheter access or direct puncture access of the aneurysmal vein and treatment with ethanol and coils, or even by coils alone. Type IIIb: Can be effectively treated as above, but can be more challenging by the vein route as more veins (not a single out-flow vein) require closure. Type IV: Can be effectively treated by transcatheter or direct puncture of the innumerable microfistulous AVFs by embolization with 50\%-50\% ethanol non-ionic contrast mixture.

Conclusion This never before reported classification system has a direct impact on determining the curative endovascular and direct puncture embolization procedures and also determines the embolic agents that will successfully treat complex AVMS in the body.

Disclosures W. Yakes: None.

\section{E-043 REVERSIBLE CEREBRAL VASOCONSTRICTION SYNDROME: NON-INVASIVE IMAGING FINDINGS AND ANGIOGRAPHIC EVALUATION}

K de Macedo Rodrigues, R Hito, D Takhtani, J Lozano, A Wakhloo, A Puri. Radiology, University of Massachusetts Medical School, Worcester, MA

\subsection{6/neurintsurg-2016-012589.115}

Purpose Reversible Cerebral Vasoconstriction Syndrome (RCVS) is a transient disorder of cerebral arterial auto regulation that may have a non-specific clinical presentation, most typically initiating as an acute episode of severe (thunderclap) headache. The clinical scenario and the non-invasive imaging

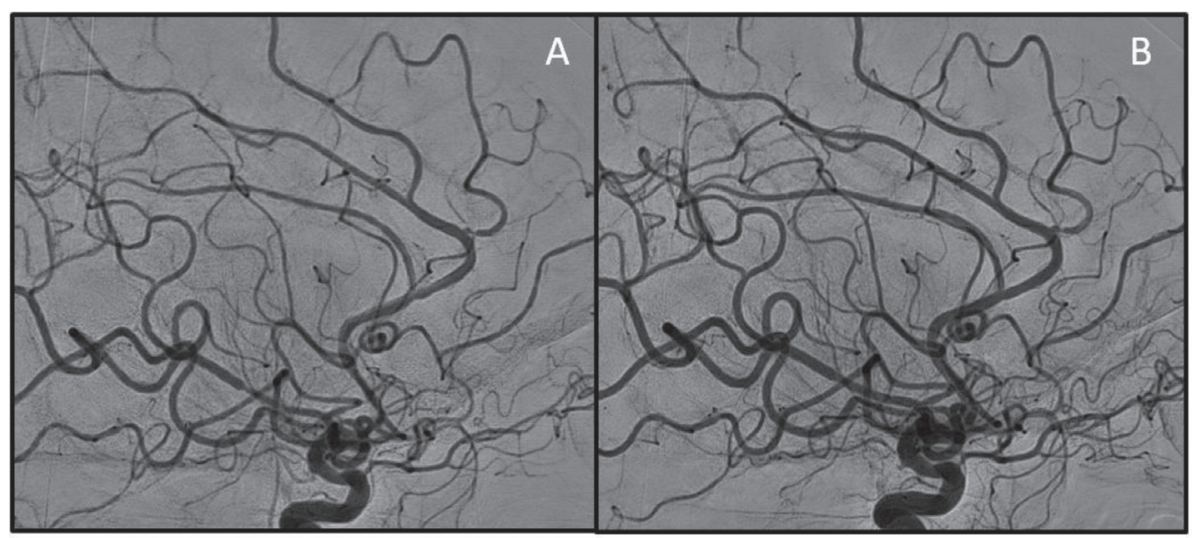

Abstract E-043 Figure 1 48-year old female presenting with severe headache and evidence of subarachnoid hemorrhage on head CT (not shown). No aneurysm or other causes for intracranial bleed was demonstrated on DSA. (A) Multiple segmentel areas of vasoconstrictions of the MCA and ACA branches are demonstrated. (B) There is significant improvement of the degree if vasoconstriction after intra-arterial administration of calcium channel blocker. 